\title{
Estrategia de gestión para mejorar la administración de los recursos públicos en la municipalidad distrital de lagunas provincia de Alto Amazonas 2021
}

\author{
Ronald Atilio Arévalo Montalván \\ Paad_26@hotmail.com \\ https://orcid.org/0000-0001-8613-0305 \\ Escuela de posgrado \\ Universidad César Vallejo \\ Hipólito Percy Barbarán Mozo \\ pbarmozito@hotmail.com \\ ORCID: 0000-0002-9316-202X \\ Escuela de Posgrado \\ Universidad César Vallejo
}

\section{RESUMEN}

El objetivo de esta revisión fue planificar la metodología de gestión para trabajar en la ordenación de los bienes públicos en la región de Lagunas - Provincia del Alto Amazonas 2021. La exploración fue claramente propositiva, no probatoria; Mientras que la población ejemplo estuvo conformada por 30 especialistas de la región distrito de Lagunas - Provincia del Alto Amazonas; La estrategia de surtido de información fue la revisión y la reunión, aplicando el Cuestionario de Estrategias de Gestión y el Cuestionario para recolectar datos sobre la variable organización de los bienes públicos. Los resultados constatan que existe un adecuado grado de ordenación, interés y competencia en el gasto en la organización de los bienes públicos de la región Lagunas - Provincia del Alto Amazonas 2021. Se comprueba que existe un grado normal de ejecución del plan de gasto de la junta (60 \%), los ejecutivos en ejecución de proyectos de especulación (70 \%) y los ejecutivos en gobierno cercano (73\%). Se concluye con la propuesta al directorio metodologías para trabajar en la ordenación de los bienes públicos en la zona de la región de Lagunas, las cuales contemplan puntos de vista que permitirán una gestión eficaz y sustentable.

Palabras clave: gestión; estrategias; recursos públicos; ejecución presupuestaria 


\title{
Management strategy to improve the administration of public resources in the district municipality of lagunas - province \\ of Alto Amazonas 2021
}

\begin{abstract}
The target of this review was to plan the administration methodology to work on the organization of public assets in the region of Lagunas - Province of Alto Amazonas 2021. The exploration was clear propositional, not trial; While the example populace was comprised of 30 specialists from the region district of Lagunas - Alto Amazonas Province; The information assortment strategy was the review and the meeting, applying the Management Strategies Questionnaire and Questionnaire to gather data on the variable organization of public assets. The results confirm that there is an adequate degree of management, interest and competition in spending in the organization of public goods in the Lagunas region - Province of Alto Amazonas 2021. It is verified that there is a normal degree of execution of the spending plan of the board (60\%), the executives in execution of speculation projects $(70 \%)$ and the executives in close government $(73 \%)$. It concludes with the proposal to the directory of methodologies to work on the management of public goods in the area of the Lagunas region, which contemplate points of view that will allow an effective and sustainable management.
\end{abstract}

Keywords: management, strategies, public resources, budget execution

Artículo recibido: 30 noviembre. 2021 Aceptado para publicación: 29 diciembre 2021

Correspondencia: Paad_26@hotmail.com Conflictos de Interés: Ninguna que declarar 


\section{INTRODUCCIÓN}

Ya existe un acuerdo entre los distintos grados de la sociedad, que el poder público es el proveedor de grandes decepciones, por su directa en administraciones o ciclos engorrosos, tacaños y de excepcionalmente baja o baja calidad; a la vez, están mal planificadas, ya que no se concentran en las necesidades más acuciantes de la población, pero además derrochan dinero y recursos humanos, lo que escandaliza a todos (Vargas y Zavaleta, 2020). En esta coyuntura, la sencillez regulatoria y la administración pública son dos temas sucesivos que se han convertido en una de las preocupaciones sociales más duraderas y que hoy se manejan en las ciencias de datos y distintas disciplinas vinculadas al espacio de la administración o gran gobierno.

En ese sentido, Suárez (2020) demostró en la ciudad de los ejecutivos, donde el acceso a los datos y la sencillez en la ejecución de proyectos abiertos y planes financieros es extremadamente restringido, ya que en su mayoría con múltiples limitaciones se llega a reportes semestrales o anuales, lo que hace mínima la viable tanto el control social como la responsabilidad (Tumi, 2020). En cualquier caso, las acciones tendientes a la pandemia de COVID-19 que han tenido en las economías evolucionadas muestran un grado más destacado que las economías nacientes, que se han visto obligadas a disminuir acciones durante las represiones importantes para confinar el virus (Khasiani et al. ., 2020).

En los países europeos, las leyes particulares sobre la sencillez y el acceso a los datos públicos se han establecido durante bastante tiempo: Noruega en 1970, Dinamarca en 1987, Finlandia en 1999 o el Reino Unido en 2000 (Rodríguez-Martín et al., 2020) y en América Latina desde principios de la década de los 90, se iniciaron cambios significativos como el intercambio de competencias de las administraciones estatales vecinales, denominadas regiones, que desglosan considerablemente su límite de directorio, lo que ha hecho que ordinariamente se llegue a circunstancias frustrantes. la realización de sus posibilidades reales en cada espacio de su ejecución (Quispe et al., 2020).

En Perú, el Ministerio de Economía y Finanzas (MEF) en 2019, precisa en la presentación de su informe anual de egresos del plan financiero por resultados, que la realización de emprendimiento público alcanzó el desarrollo del 66\% al finalizar el último día hábil del año, siendo el barrio administraciones estatales que gastaron $\mathrm{S} / 12,416$ millones, $14.8 \%$ menos en comparación con 2018. Es menester puntualizar que el ejercicio con principal 
gasto se encuentran en la dinámica interna local y externa nacional con (61.9\%), desinfección con $(57.2 \%)$, capacitación y fortalecimiento de capacidades (66.7\%). y horticultura (65,5\%), (Rodríguez et al., 2020).

Los residentes piden franqueza en las actividades de implementación de políticas y en la actividad de la fuerza, durante bastante tiempo, dado que es probable que las administraciones estatales basadas en la popularidad brinden franqueza en sus actividades, ya que es la regla esencial de la tipo de gobierno. Según la famosa representación (Labandeira, 2020), existen instrumentos legítimos para la consistencia con el acceso a los datos, así como varios resultados concebibles presentados por la web para el comercio de datos que han avanzado cambios en el entorno informático (López, et al, 2016, p. . 28 ). Las ramificaciones de esta abundancia de datos han producido peculiaridades impulsadas por la información, con las cuales las líneas del patrón son claras: índices de información de enorme alcance y su variedad, investigación y traducción son progresivamente esenciales para descubrir y obtener estima. los datos (Guaycha y Ordóñez, 2020).

La sencillez en los temas legislativos es solicitada con mucho más entusiasmo, por grupos ideológicos y además por vecinos, que por fin ayudan con sus valoraciones a las condiciones de los actuales gobiernos populares en las naciones donde existen. El interés social por la sencillez se articula idealmente hacia las organizaciones públicas, ya que éstas supervisan los bienes públicos y establecen decisiones que afectan a toda la población que habita en un país, región o en otros contornos regionales (Ruano y HerreroGutiérrez, 2020). Debe advertirse que las regiones comunales y de área son los órganos administradores que promueven el mejoramiento de los barrios, con personería jurídica de derecho abierto y plena capacidad para satisfacer sus motivaciones.

A su vez, las normas vecinales aprecian la independencia política, financiera y autoritaria en temas de su competencia (Cárdenas, 2019) y enfrentan demandas crecientes con respecto a los vecinos, especialmente en cuanto a la forma de conexión, que van desde la disposición de administraciones convencionales, por ejemplo, reaccionando a sistemas y preocupaciones, reforzando el método de interrelación entre ambos. Esta necesidad ha sido impulsada y trabajada, en general, por la utilización de aparatos avanzados y avances de datos y correspondencia, dando lugar a la cosmovisión de Gobierno Abierto (ToroGarcía et al., 2020). 
Debe señalarse que las municipalidades que integran la provincia de Alto Amazonas presentan grandes desafíos como respuesta a los objetivos de servicio público, que deben ser ejecutados con la mejor claridad, fundamentalmente que sus servidores actúen con transparencia, es importante acotar que la eficiencia en la dirección de los recursos públicos son valores que deben integrar las labores del servidor público, estos desafíos resulta al definir los objetivos claros y sencillos que informen las inquietudes institucionales, y transparenten para que la imagen social institucional sea adecuada. Cabe recalcar que la gestión de la inteligencia es otra competencia que debemos cultivar para acceder a buenas acciones y actitudes estratégicas, todo ello necesita de una gran labor en lo que refiere al conocimiento de la realidad y que sea manejable y elástico a los cambios. Para un buen despunte de nuestra gestión es necesario conocer la focalización de los hechos con amplitud y profundidad, teniendo en consideración la creación de valor institucional todo es posible para luego dar a la sociedad.

Como punto final la competencia de capacidades y autorregulación, implica supervisar el sistema y coordenadas del régimen organizacional para ejecutar los ajustes necesarios como requisito para hacer crecer la institución entre ellos el proceso laboral y de la administración de los recursos públicos, como parte integrante de un escenario de mejora continua. En ese sentido esta investigación tiene como propósito diseñar estrategias para una adecuada dirección institucional, a fin de mejorar la transparencia en la administración de los recursos públicos en la Municipalidad Distrital de Lagunas Provincia de Alto Amazonas - Región Loreto.

\section{Frente a ello, surge el problema de investigación:}

- ¿De qué manera la estrategia de gestión mejorará la administración de los recursos públicos en la municipalidad distrital de Lagunas - Provincia de Alto Amazonas 2021?, y

\section{Los problemas específicos}

a) ¿Cuáles son las estrategias de gestión utilizadas en la administración de los recursos públicos en la municipalidad distrital de Lagunas - Provincia de Alto Amazonas 2021?,

b) b)¿Cuáles son las características utilizadas en la gestión de los recursos públicos en la municipalidad distrital de Lagunas - Provincia de Alto Amazonas 2021? y, 
c) ¿Cuál es el grado de validez de la estrategia de gestión para mejorar la administración de los recursos públicos en la municipalidad distrital de Lagunas - Provincia de Alto Amazonas 2021?

Se justifica por conveniencia, dado la repercusión de las acciones relacionadas a la transparencia, entendida como el comportamiento de la administración pública a la voluntad del ciudadano, permitiendo una mayor supervisión y fiscalización de la sociedad a las municipalidades, formulación y evaluación de las políticas establecidas por ley. Así, la ejecución de las metodologías del tablero sugiere que cada una de sus partes se aplique a cada clase fundamental del elemento, convirtiéndose en un ciclo siempre coordinado y dinámico; Como primer avance, cada elemento debe configurar los destinos, enfoques y sistemas conectados entre sí, para asegurar el giro jerárquico de los acontecimientos y la satisfacción de los objetivos corporativos.

En consecuencia, se defiende el trabajo de exploración actual de acuerdo con las perspectivas que lo acompañan. La relevancia social será una fuente de consejo para que la investigación futura se concentre en los factores, de modo que afectará el beneficio de los estudiantes universitarios, educadores y estudiantes de doctorado, así como los maestros reales y la población general, así como el número de habitantes en el Distrito de Lagunas - Provincia del Alto Amazonas-Región Loreto, a la que se inclina, en virtud de que la revisión permitió avanzar en la respetabilidad, franqueza y obligación en la función pública, garantizando la correcta presentación de sus capacidades en la organización de bienes públicos. El valor teórico; se fundó explícitamente en liderar un estudio interno y externo sobre la rectitud en la organización de los bienes públicos; por lo que los resultados adquiridos se suman al universo epistemológico de la ciencia; así como la investigación de arriba a abajo de las especulaciones que lo componen.

Por su parte, la implicancia practica; está en los resultados obtenidos, ya que permitieron proponer procedimientos para sumar a la atención de los problemas rastreados en función de la franqueza en la organización de los bienes públicos en la comuna de Lagunas, zona Loreto 2020"; así que se han sumado a dar las metodologías esenciales para ordenar y administrar bien los bienes del Estado y de esta manera que los pueblos de este pedazo de la nación cumplan con su anhelado giro económico y social. La utilidad metodológica de las estrategias, metodología, procedimientos e instrumentos utilizados en la exploración, cuando se haya ilustrado su legitimidad y calidad inquebrantable, podrá ser 
utilizada en otros trabajos de exploración; El instrumento aplicado es la encuesta, la cual nos permitió conocer la verdad de la franqueza en la organización de los bienes públicos en la localidad comuna de Lagunas zona Loreto 2021.

Se planteó como objetivo general Proponer estrategias de gestión para mejorar la administración de los recursos públicos en la municipalidad distrital de Lagunas Provincia de Alto Amazonas 2021.

\section{Siendo los objetivos específicos:}

- Caracterizar las estrategias de gestión utilizadas en la administración de los recursos públicos en la municipalidad distrital de Lagunas - Provincia de Alto Amazonas 2021 ,

- Describir las características utilizadas en la gestión de los recursos públicos en la municipalidad distrital de Lagunas - Provincia de Alto Amazonas 2021 y

- Validar estrategia de gestión para mejorar la administración de los recursos públicos en la municipalidad distrital de Lagunas - Provincia de Alto Amazonas 2021. Y se busca comprobar la hipótesis: Si aplicamos las estrategias de gestión entonces se podrá mejorar la administración de los recursos públicos que caracteriza a la Municipalidad Distrital de Lagunas - Provincia de Alto Amazonas- Región Loreto, año 2020.

\section{METODOLOGÍA}

\section{Tipo de estudio}

El examen actual es ciencia pura o fundamental, ya que consiste en buscar información sobre las peculiaridades, retratarlas, esclarecerlas y anticiparlas (Sánchez et al., 2018). Del mismo modo, es de tipo descriptivo propositivo; por la elaboración de un modelo o proposición de respuesta pragmática para una necesidad o problema de un establecimiento o reunión; a partir de una determinada determinación de necesidades en un determinado segundo así como de los ciclos productivos o lógicos que intervienen y patrones futuros, es decir, se completa en función de las consecuencias de una interacción de evaluación previa (Hurtado, 2000).

Lo propositivo, comprende la elaboración de una proposición o modelo, como respuesta a un problema o necesidad de tipo funcional, ya sea de una reunión, o de una organización, a la luz de una determinación exacta de las necesidades existentes al margen de todo lo 
demás, los ciclos lógicos o generativos incluidos. es más, patrones futuros, eso, depende de las consecuencias de una interacción perspicaz (Hurtado, 2000).

El tipo de técnica que se seguirá para lograr los objetivos propuestos se relaciona con el examen No Experimental, ya que no cambiará deliberadamente las partes de ninguno de los factores. Bajo esta metodología no exploratoria, el plan adecuado para nuestro examen es el Transversal. Ya que recopilaremos la información en un tiempo único, para describir los factores de revisión y examinar su frecuencia (Juárez, 2014).

Es de carácter descriptivo; porque tiene como objetivo indagar la incidencia y valores manifestada en una o más variables para luego proporcionar su descripción (Juarez, 2014).

Se esquematiza por el siguiente diseño:

Dónde:

$\mathbf{R x}=$ Realidad diagnosticada.

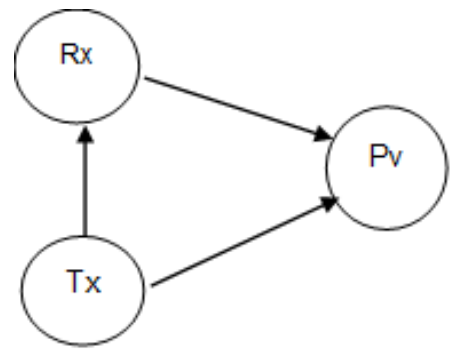

$\mathbf{T x}=$ Teorías sobre estrategia de gestión

$\mathbf{P v}=$ Modelo de propuesta validada

\subsection{Variables y operacionalización.}

\section{Variable: Estrategia de gestión}

Definición conceptual: Vincula la asignación de recursos a productos y resultados medibles a favor de la población y es fundamental para alcanzar el logro de los objetivos institucionales, haciendo uso de los recursos humanos capacitados (Secretaría de Asuntos Políticos et al., 2012).

Definición operacional: Se utilizara como herramienta o instrumento el cuestionario y una escala tipo Lickert, en base a los factores, en consecuencia se podrá implementar las estrategias de gestión.

\section{Dimensión:}

- Planificación.

- Participación.

- Eficiencia y capacidad de gasto. 


\section{Variable: Administración de los recursos públicos}

Definición conceptual: Es la fusión de la orientación normativa de la administración pública tradicional y de la orientación instrumental de la gestión en sentido genérico (Valeriano Ortiz, 2013).

Definición operacional: Es la manifestación de la administración a través de la ejecución presupuestal, Ejecución de proyectos de inversión, consulta de transparencias a los gobiernos locales, asimismo consulta de la deuda pública interna y externa.

\section{Dimensión:}

- Seguimiento de la ejecución presupuestal

- Ejecución de proyectos de inversión.

- Consulta de transparencia.

- Consulta de la deuda pública interna y externa (Ver: Anexo1: Matriz de operacionalización).

\subsection{Población}

\section{Población}

- Estuvo conformada por 06 Municipalidades distritales de la Provincia de Alto Amazonas, Región Loreto 2021.

\section{Muestra}

- Fueron 30 trabajadores de la Municipalidad distrital de lagunas, que integra toda la organización administrativa.

\section{Muestreo}

- El muestreo fue aleatorio estratificado.

\section{Criterios por exclusión}

- Trabajadores que al momento de la aplicación del instrumento no se encontraron en su centro laboral

\subsection{Técnicas e instrumentos de recolección de datos, validez y confiabilidad.}

\section{Técnica}

- Se utilizó la técnica de la encuesta y el análisis de documentos.

\section{Instrumento}

Se utilizó el cuestionario con el fin de conocer el manejo administrativo de los fondos públicos de información directa de los trabajadores de la entidad municipal "Municipalidad Distrital de lagunas". También se empleó el fichaje de texto, para la 
estructuración del marco teórico, a través de libros, tesis, artículos científicos, monografías, agregando revistas especializadas.

\section{Validez}

Para proceder a la aprobación de los instrumentos se utilizó el método del juicio maestro. La elección de los especialistas se realizó deliberada y básicamente, en consecuencia, se determinó la apertura a la población, su accesibilidad e interés en ser importantes para esta revisión, su imparcialidad, libertad e involucramiento en el campo de la implementación de políticas (Zamora-de -Ortiz et al., 2020).

La legitimidad de fondo se ayudó a través del "juicio maestro", que busca relevar las consultas según las medidas de trascendencia, pertinencia y claridad, así como investigar la redacción y que sean razonables por la población a la que se dirige. (Mezarina et al., 2021).

Se tuvo en consideración 05 expertos y teniendo como resultado lo presentado en la siguiente tabla:

Tabla 1.

Validación por criterio de expertos

\begin{tabular}{|c|c|c|c|c|c|}
\hline Variable & $\mathbf{N}^{\mathbf{o}}$ & $\begin{array}{c}\text { Experto o } \\
\text { especialista }\end{array}$ & $\begin{array}{l}\text { Promedio } \\
\text { de validez }\end{array}$ & $\begin{array}{l}\text { porcentaje } \\
\text { de validez }\end{array}$ & $\begin{array}{l}\text { Opinión del } \\
\text { experto }\end{array}$ \\
\hline \multirow{5}{*}{$\begin{array}{l}\text { Estrategias } \\
\text { de gestión }\end{array}$} & 1 & $\begin{array}{l}\text { Valles Coral Miguel } \\
\text { Ángel }\end{array}$ & 48 & $96.00 \%$ & $\begin{array}{c}\text { Existe } \\
\text { consistencia }\end{array}$ \\
\hline & 2 & $\begin{array}{lll}\text { Juarez } & \text { Díaz } & \text { Juan } \\
\text { Rafael } & & \\
\end{array}$ & 49 & $98.00 \%$ & Es aplicable \\
\hline & 3 & $\begin{array}{l}\text { Ríos } \\
\text { Orlando }\end{array}$ & 50 & $100.00 \%$ & $\begin{array}{c}\text { Con } \\
\text { consistencia }\end{array}$ \\
\hline & 4 & $\begin{array}{l}\text { Bardales Zapata } \\
\text { Efraín de la Cruz }\end{array}$ & 48 & $96.00 \%$ & $\begin{array}{l}\text { Es pertinente } \\
\text { y coherente }\end{array}$ \\
\hline & 5 & $\begin{array}{l}\text { Barzola Cárdenas } \\
\text { Abner Milán }\end{array}$ & 48 & $96.00 \%$ & $\begin{array}{l}\text { Coherencia } \\
\text { metodológica }\end{array}$ \\
\hline \multirow{5}{*}{$\begin{array}{l}\text { Administrac } \\
\text { ión de los } \\
\text { recursos } \\
\text { públicos }\end{array}$} & 1 & $\begin{array}{l}\text { Valles Coral Miguel } \\
\text { Ángel }\end{array}$ & 48 & $96.00 \%$ & $\begin{array}{c}\text { Con } \\
\text { consistencia } \\
\end{array}$ \\
\hline & 2 & $\begin{array}{lll}\text { Juarez } & \text { Díaz } & \text { Juan } \\
\text { Rafael } & & \\
\end{array}$ & 48 & $96.00 \%$ & Es aplicable \\
\hline & 3 & $\begin{array}{l}\text { Ríos } \\
\text { Orlando }\end{array}$ & 49 & $98.00 \%$ & Consistente \\
\hline & 4 & $\begin{array}{l}\text { Bardalez Zapata } \\
\text { Efraín de la Cruz }\end{array}$ & 48 & $96.00 \%$ & $\begin{array}{c}\text { Pertinente y } \\
\text { coherente }\end{array}$ \\
\hline & 5 & $\begin{array}{l}\text { Barzola Cárdenas } \\
\text { Abner Milán }\end{array}$ & 48 & $96.00 \%$ & $\begin{array}{c}\text { Suficiencia y } \\
\text { coherencia }\end{array}$ \\
\hline
\end{tabular}


Los instrumentos, que consisten en dos cuestionarios, fueron sometidos al juicio de los expertos mencionados en el cuadro anterior; quienes efectuaron la verificación de la coherencia y pertinencia de los indicadores con las variables. En cuanto a la primera variable, el resultado arrojó un promedio de 4.86, representando el 97.20\% de concordancia entre jueces. Respecto a la segunda variable, arrojó un promedio de 4.82, representando el $96.4 \%$ de concordancia entre jueces; lo que indica, que tienen alta validez; reuniendo las condiciones metodológicas para ser aplicado.

\section{Confiabilidad}

La confiabilidad indica el valor de alfa de Cronbach, para el presente estudio es de 9.57, lo que indica un alto nivel de confiabilidad.

\subsection{Procedimientos}

Para la fase diagnóstica; se hizo un diagnóstico del contexto situacional; identificándose el problema más saltante del contexto; así mismo, se exploró la realidad problemática de manera general hasta llegar a la realidad focalizada; formulándose los problemas y objetivos. Se recopiló información para implementar los antecedentes y el marco teórico. También se eligió el diseño a utilizar, se determinó la muestra y los instrumentos de recolección de información. Los resultados obtenidos fueron sometidos a discusión y conllevaron a las conclusiones respectivamente.

Para la fase diseño; se buscaron alternativas de solución al problema encontrado, a través de propuestas viables. Realizándose la revisión de estrategias de gestión de los cambios vigentes y de cómo estos han contribuido en la solución de problemas en las organizaciones. Asimismo, se revisaron las teorías que ayudan a profundizar el conocimiento sobre estas estrategias.

La fase validación; permitió dar la prestancia y garantizar la aplicabilidad de la estrategia de salud pública; cambio que fue sometido a juicio de expertos para su validación respectiva. Finalmente, la estrategia de gestión propuesta se sustentó y socializó.

\subsection{Métodos de análisis de datos}

Una vez recolectada la información mediante la aplicación del cuestionario se procederá a la revisión y codificación de la misma para organizarla y facilitar el proceso de tabulación. Se procederá a la categorización con la finalidad de que cada pregunta tenga los grupos y clases necesarias para su respuesta y de esta manera facilitar la tabulación de la información. 
La tabulación se realizó de forma manual ya que es un número reducido de datos y se utilizará para su tabulación es sistema quinario, para el análisis de los datos se utilizará la investigación descriptiva seleccionando el estadígrafo de los porcentajes y para la presentación de la información se utilizará la presentación de datos semitabular EXCEL para poder interpretar con mayor claridad la investigación realizada.

\subsection{Aspectos éticos}

Nuestro estudio de investigación, se fundamenta en el respeto al conocimiento y a la calidad del proceso de investigación, a las personas que intervinieron activamente en ella debido a la confidencialidad y reserva de sus datos personales y también en las personas a quienes el contenido de la misma puede servir como referente, debido a lo escrupuloso de la obtención y encausamiento de la indagación, fundamentados en principios benéficos En estas formulaciones sobre la esencia del buen gobierno y de la buena administración, suele estar siempre presente la dimensión ética, seguramente porque se ha entrado en razón de que el gobierno y la dirección de las instituciones públicas no son una actividad neutral. Muy por el contrario, debe estar orientada al bienestar integral de los ciudadanos y debe facilitar, por tanto, a quienes la realiza su mejoramiento personal. La dimensión ética incorpora un componente esencial al buen gobierno y la buena administración: el objetivo que toda persona debe perseguir es el bien; principio en cuya virtud, tanto el personal al servicio de la administración pública como los ciudadanos, han de actuar con honestidad, rectitud y lealtad. La razón debe buscarse en el grado de compromiso real de los gobernantes y administradores con la mejora de las condiciones de vida de los ciudadanos. El servicio objetivo al interés general implica entender preferentemente las necesidades colectivas de las personas.

El buen gobierno y la buena administración tienen mucho que ver con la adecuada preparación de las personas que la dirijan. Es necesaria una mentalidad abierta y sensibilidad social; deben trabajar sobre la realidad, ser capaces de contemplar y razonar acerca de los problemas colectivos desde perspectivas de equilibrio, a fin de lograr comprender los problemas y contemplar la pluralidad de enfoques y dimensiones que encierran. Por otra parte, si bien los empleados públicos necesitan de los medios y condiciones de trabajo adecuados, para poder desempeñar su tarea con eficacia, deben brindar un constante esfuerzo en su competencia profesional. El concepto de buena administración es un concepto se proyecta sobre los responsables, sobre los funcionarios. 
Es posible constituir grandes teorías sobre la buena administración en el aire, pero la mejor teoría sobre la materia es un buen directivo o gobernante en acción.

En consecuencia, desde la perspectiva ética del servicio público, se establecen unos principios básicos de objetividad, integridad, neutralidad, responsabilidad, credibilidad, imparcialidad, confidencialidad, dedicación al servicio público, transparencia, ejemplaridad, austeridad, accesibilidad, eficacia, honradez y promoción del entorno cultural y medioambiental, y de la igualdad entre hombres y mujeres; que delimitan el contenido del concepto de buen gobierno y buena administración desde el plano ético.

\section{RESULTADOS}

a) Caracterizar las estratégias de gestión utilizadas en la administración de los recursos públicos en la municipalidad distrital de Lagunas - Provincia de Alto Amazonas 2021.

Tabla 2. Nivel de gestión en planificación

\begin{tabular}{ccc}
\hline Niveles de gestión en planificación & Frecuencia & Porcentaje \\
\hline Deficiente & 02 & $6.70 \%$ \\
\hline Aceptable & 19 & $63.30 \%$ \\
\hline Eficiente & 9 & $30.00 \%$ \\
\hline Total & $\mathbf{3 0}$ & $\mathbf{1 0 0 . 0 0 \%}$ \\
\hline
\end{tabular}

Fuente: Elaboración propia

En la tabla 02 podemos observar que existe un nivel aceptable de planificación en la administración de recursos públicos, que está presente en el 63.30\% (19) de los empleados de la municipalidad distrital de Lagunas - Provincia de Alto Amazonas 2021, y a su vez nos muestra que el $6.70 \% \%$ (02) identifican un nivel deficiente, lo que indica una percepción negativa o nula del proceso de planificación. Sin embargo, debemos mencionar que un porcentaje importante de los trabajadores (30.00\%), señalan que consideran que la gestión de la planificación es eficiente.

Tabla 3. Nivel de gestión en participación

\begin{tabular}{ccc}
\hline Niveles de gestión en participación & Frecuencia & Porcentaje \\
\hline Deficiente & 01 & $3.30 \%$ \\
\hline Aceptable & 20 & $66.70 \%$ \\
\hline Eficiente & 09 & $30.00 \%$ \\
\hline Total & $\mathbf{3 0}$ & $\mathbf{1 0 0 . 0 0 \%}$ \\
\hline
\end{tabular}

Fuente: Elaboración propia 
En la tabla 03 podemos observar que existe un nivel aceptable de la participación en la administración de recursos públicos, que está presente en el 66.70\% (20) de los empleados de la municipalidad distrital de Lagunas - Provincia de Alto Amazonas 2021, y a su vez nos muestra que el 3.30\% (01) señalan un nivel deficiente. Sin embargo, debemos mencionar que un porcentaje importante de los trabajadores $(30.00 \%)$, señalan que consideran que la gestión de la participación es eficiente. En esta dimensión podemos señalar que existe mayor convicción sobre el sentimiento de participación en la gestión.

Tabla 4. Nivel de gestión en eficiencia y capacidad de gasto

\begin{tabular}{ccc}
\hline Niveles de gestión en eficiencia y capacidad de gasto & Frecuencia & Porcentaje \\
\hline Deficiente & 04 & $13.30 \%$ \\
\hline Aceptable & 15 & $50.00 \%$ \\
\hline Eficiente & 11 & $36.70 \%$ \\
\hline Total & $\mathbf{3 0}$ & $\mathbf{1 0 0 . 0 0 \%}$ \\
\hline
\end{tabular}

Fuente: Elaboración propia

En la tabla 04, podemos observar que existe un nivel aceptable de la eficiencia y capacidad de gasto en la administración de recursos públicos, que está presente en el $50.00 \%$ (15) de los empleados de la municipalidad distrital de Lagunas - Provincia de Alto Amazonas 2021, y a su vez nos muestra que el $13.30 \%$ (04), señalan que la capacidad de gasto es deficiente. Sin embargo, debemos mencionar que un porcentaje de los trabajadores $(36.70 \%)$, señalan que consideran que la gestión en eficiencia y capacidad de gasto es eficiente. En esta dimensión podemos señalar que existe mayor contradicción en la dimensión eficiencia y capacidad de gasto.

a) Identificar y describir las características utilizadas en la gestión de los recursos públicos en la municipalidad distrital de Lagunas - Provincia de Alto Amazonas 2021.

Tabla 5. Nivel de gestión en ejecución presupuestaria

\begin{tabular}{ccc}
\hline Niveles de gestión en ejecución presupuestaria & Frecuencia & Porcentaje \\
\hline Malo & 03 & $10.00 \%$ \\
\hline Regular & 18 & $60.00 \%$ \\
\hline Bueno & 01 & $3.30 \%$ \\
\hline Excelente & 08 & $26.70 \%$ \\
\hline Total & $\mathbf{3 0}$ & $\mathbf{1 0 0 . 0 0 \%}$ \\
\hline
\end{tabular}

Fuente: Elaboración propia

En la tabla 05, podemos observar que existe un nivel de gestión en ejecución presupuestaria regular, en un $60 \%$ (18) de los recursos públicos en la municipalidad 
distrital de Lagunas - Provincia de Alto Amazonas 2021, y a su vez nos muestra que el $10.00 \%(03)$ se encuentra en un nivel malo, lo que indica que existe escasa ejecución presupuestaria aun cuando cuentan con un proceso de planificación.

Tabla 6.

Nivel de gestión en ejecución de proyectos de inversión

\begin{tabular}{ccc}
\hline Niveles de gestión en ejecución de proyectos de inversión & Frecuencia & Porcentaje \\
\hline Malo & 01 & $03.30 \%$ \\
\hline Regular & 21 & $70.00 \%$ \\
\hline Bueno & 02 & $6.70 \%$ \\
\hline Excelente & 06 & $20.00 \%$ \\
\hline Total & $\mathbf{3 0}$ & $\mathbf{1 0 0 . 0 0 \%}$ \\
\hline
\end{tabular}

Fuente: Elaboración propia

En la tabla 06, podemos observar que existe un nivel regular de gestión en ejecución de proyectos de inversión, que está señala el 70.00\% (21) de trabajadores en la gestión de los recursos públicos en la municipalidad distrital de Lagunas - Provincia de Alto Amazonas 2021, y a su vez nos muestra que el $20.00 \%$ (06) señalan un nivel excelente, demostrando dos posiciones contrarias en relación a la percepción de la ejecución de proyectos.

Tabla 7. Nivel de gestión a los gobiernos locales

\begin{tabular}{ccc}
\hline Niveles de gestión a los gobiernos locales & Frecuencia & Porcentaje \\
\hline Malo & 00 & $00.00 \%$ \\
\hline Regular & 22 & $73.30 \%$ \\
\hline Bueno & 01 & $3.30 \%$ \\
\hline Excelente & 07 & $23.30 \%$ \\
\hline Total & $\mathbf{3 0}$ & $\mathbf{1 0 0 . 0 0 \%}$ \\
\hline
\end{tabular}

Fuente: Elaboración propia

En la tabla 07 podemos observar que el $73 \%$ de trabajadores de la municipalidad de Lagunas - Provincia de Alto Amazonas señala que la gestión en el gobierno local es de nivel regular, y a su vez nos muestra que el $23.30 \%$ (07) manifiestan un nivel excelente, lo que indica que preponderantemente hay una percepción de limitada gestión en el gasto público de los gobiernos locales, condiciones que en el tiempo derivan en un problema presupuestal. 
Tabla 8 .

Nivel de gestión en consulta de deuda

\begin{tabular}{ccc}
\hline Niveles de gestión en consulta de deuda & Frecuencia & Porcentaje \\
\hline Malo & 01 & $03.30 \%$ \\
\hline Regular & 21 & $70.00 \%$ \\
\hline Bueno & 03 & $10.00 \%$ \\
\hline Excelente & 05 & $16.70 \%$ \\
\hline Total & $\mathbf{3 0}$ & $\mathbf{1 0 0 . 0 0 \%}$ \\
\hline
\end{tabular}

Fuente: Elaboración propia

En la tabla 08 podemos identificar que el nivel regular de gestión en consulta de deuda en la municipalidad de Lagunas - Provincia de Alto Amazonas, presenta un porcentaje elevado que es igual a 70.00\%, evidenciando la escasa atención que se brinda a este problema.

c) Validar estrategias de gestión para mejorar la administración de los recursos públicos en la municipalidad distrital de Lagunas - Provincia de Alto Amazonas 2021.

En seguida se estableció el proceso de validez de la propuesta

Tabla 9. Validez de la propuesta.

\begin{tabular}{|c|c|c|c|c|c|c|c|c|}
\hline \multirow[b]{2}{*}{ Criterios } & \multicolumn{5}{|c|}{ Jueces } & \multirow[b]{2}{*}{ Sumatoria } & \multirow[b]{2}{*}{ Promedio } & \multirow[b]{2}{*}{ Porcentaje } \\
\hline & $\begin{array}{c}\text { Juez } \\
01\end{array}$ & $\begin{array}{c}\text { Juez } \\
\text { 02 }\end{array}$ & $\begin{array}{c}\text { Juez } \\
\mathbf{0 3}\end{array}$ & $\begin{array}{c}\text { Juez } \\
\text { 04 }\end{array}$ & $\begin{array}{c}\text { Juez } \\
\text { 05 }\end{array}$ & & & \\
\hline $\begin{array}{llr}\text { Los } & \text { objetivos } & \text { son } \\
\text { coherentes con la propuesta }\end{array}$ & 4 & 4 & 5 & 5 & 5 & 23 & 4,6 & $92 \%$ \\
\hline $\begin{array}{l}\text { La metodología es la } \\
\text { adecuada para lograr los } \\
\text { objetivos }\end{array}$ & 5 & 4 & 5 & 4 & 4 & 22 & 4,4 & $88 \%$ \\
\hline $\begin{array}{l}\text { Presenta } \quad \text { aspectos } \\
\text { innovadores en su proceso }\end{array}$ & 4 & 4 & 4 & 4 & 4 & 20 & 4,0 & $80 \%$ \\
\hline $\begin{array}{l}\text { El procedimiento es el } \\
\text { adecuado para la población }\end{array}$ & 4 & 5 & 4 & 5 & 4 & 22 & 4,4 & $88 \%$ \\
\hline $\begin{array}{ll}\text { Los contenidos } & \text { son } \\
\text { pertinentes } & \end{array}$ & 5 & 5 & 5 & 5 & 5 & 25 & 5,0 & $100 \%$ \\
\hline $\begin{array}{l}\text { Los pasos a seguir son } \\
\text { realizables }\end{array}$ & 4 & 4 & 4 & 4 & 4 & 20 & 4,0 & $80 \%$ \\
\hline $\begin{array}{l}\text { La implementación } \\
\text { presenta aspectos de } \\
\text { sostenibilidad y viabilidad }\end{array}$ & 5 & 5 & 5 & 5 & 5 & 25 & 6,00 & $100 \%$ \\
\hline
\end{tabular}

Fuente: Elaboración propia 
Consistió en la elaboración de la guía y escala. La guía elaborada consta de 18 preguntas y 07 aspectos (dimensiones, fundamentación teórica, operatividad y propuesta), la escala de valoración está compuesta de cinco categorías y se incluyen ítem de selección sobre los criterios, puntos de vista y argumentos, de cada aspecto del resultado, que fue sometida a consideración de los expertos.

- Coherencia de los objetivos de la propuesta

- Adecuación de la metodología planteada frente a los objetivos propuestos

- Aspectos innovadores de la metodología

- Adecuación del procedimiento para el tipo de población

- Pertinencia de los contenidos para el tipo de estrategia

- Realización de los pasos a seguir

- Implementación con sostenibilidad y viabilidad

Los resultados de la evaluación estrategias de gestión para mejorar la administración de los recursos públicos en la municipalidad distrital de Lagunas - Provincia de Alto Amazonas 2021, realizada por los expertos, son de significación estadística, es decir, hay evidencias suficientes para plantear, a un $95 \%$ de confiabilidad, que los 5 expertos concuerdan en que las estrategias en la administración de los recursos públicos en la municipalidad, es acertado, tanto en su concepción teórica como en los resultados que se obtendrán con su aplicación como actividad en el proceso de gestión.

\section{DISCUSIÓN}

Se encontró un nivel aceptable de planificación en la administración de recursos públicos, que está presente en el 63.30\% (19), en participación se encontró un nivel aceptable en el $66.70 \%$ (20) y el 3.30\% (01) señalan un nivel deficiente. En relación a la eficiencia y capacidad de gasto en la administración de recursos públicos, existe un nivel aceptable en el $50.00 \%$ (15), a su vez nos muestra que el $13.30 \%$ (04), señalan que la capacidad de gasto es deficiente de los empleados de la municipalidad distrital de Lagunas - Provincia de Alto Amazonas 2021. Por su parte, Alarcón et al. (2020), señala que el grado de validez que llegan los gobiernos de las localidades se diferencian por dimensiones claves que describen la actividades de gestión, asimismo estas actividades se utilizan como base para realizar exámenes de desempeño a los servidores públicos. Estas actividades de implementación es ejecutado por la administración municipal Mayarí, en la provincia Holguín, pueblo ubicado al interior del oriente cubano. Estableciendo asimismo 
dimensiones exactas y claves que resuelven con claridad y efectividad las actividades realizadas interna y externamente en la administración gubernamental, siempre con la asesoría y aplicando técnicas, que califican de manera efectiva los niveles del gobierno, sin divorciarse del apoyo estadístico. Por otra parte, Cardenas (2019), demuestran que no existe incidencia de las estrategias de gestión municipal en el desarrollo organizacional de la Municipalidad provincial de Otuzco 2018. Además, Guzmán (2020), Resalta que la administración Municipal Distrital de La Banda de Shilcayo con un nivel "Regular" de treinta y nueve por ciento, y la aplicación de técnicas estratégicas frente al virus letal que afecta el país en general, en nuestro caso al distrito de la banda de shilcayo, nos informa un nivel "Regular" con cincuenta por ciento; de la misma manera, existe una conexión alta positiva entre la gestión administrativa municipal y las estrategias aplicadas frente al virus covid 19 en la municipalidad Distrital - Banda de Shilcayo, 2020. Expresando que ochenta y cuatro por ciento en la organización edil, puesta en marcha las estrategias frente al Covid-19 es influenciado por la administración municipal distrital de La Banda de Shilcayo. A su vez, Flores (2021), los resultados determinaron que el nivel de efectividad de la mejora continua en la Municipalidad distrital de Morales, 2020, muestra un nivel casi siempre con 37\%. Asimismo, el nivel de transparencia de gestión en la Municipalidad distrital de Morales, 2020, tiene un nivel a veces con un 42\%; concluyendo que existe relación entre la mejora continua y transparencia de gestión en la Municipalidad Distrital de Morales, 2020. También, Quispe et al. (2020), Obteniendo como resultado que el $97.3 \%$ de los ciudadanos perciben una baja calidad de la gestión municipal y solo un $2.7 \%$ percibieron una mediana calidad, resultando oportuna la implementación de un modelo de open government que incluya un conjunto de estrategias y acciones basadas en la transparencia y acceso a la información pública, participación y colaboración ciudadana, rendición de cuentas e integridad, innovación y tecnología, que permita optimizar la gestión municipal. Por su parte, Rodriguez et al., (2020), logró establecer que existe un nivel bajo del gasto público desde la percepción del trabajador en un $87.5 \%$ y del funcionario en $80 \%$.

Se observa un nivel de gestión en ejecución presupuestaria regular, en un 60\%, en ejecución de proyectos de inversión, observamos un nivel regular en el 70.00\%, en la gestión del gobierno local se observa el nivel regular con 73\%, y en la gestión en consulta de deuda se encontró el nivel regular con $70.00 \%$ en la municipalidad de Lagunas - 
Provincia de Alto Amazonas. En ese sentido, Molina et al. (2018), Los resultados señalan que el Ayuntamiento de Barcelona tiene dificultades para realizar un correcto seguimiento de la transparencia de las entidades. En las conclusiones se propone la creación de estruturas compartidas de recursos y estratégias de formación y sensibilización para salvar la falta de tradición y conocimiento que padecen tanto las entidades como las administraciones públicas locales. También Rivera (2020), encontro que las actividades actuales en lo que refiere a la utilización de herramientas de gestión relacionados con la transparencia en las cinco administraciones municipales en la Provincia de Mariscal Cáceres, 2018, ello, relacionado con dimensiones y seguimiento del cumplimiento presupuestal y la ejecución de los proyectos de inversión, consultas de las transferencias y obligaciones corrientes y no corrientes, obligaciones interna y externa; es regular, y el grado o nível de ejecución presupuestaria, en las dimensiones programación de compromisos anual, certificación, ejecución y gastos presenta un nivel medio. A su vez, Tumi (2020), el proceso rendible de cuentas del gobierno municipal de Puno implementado a través de las audiencias públicas centralizadas o descentralizadas, tuvo un carácter parcial, agregado y limitada representatividad. Por otro lado, Vargas \& Zavaleta (2020), encontró que la ejecución alcanzada por los 83 distritos cuya ejecución alcanza sólo un 66\%, habiendo medido la calidad del gasto, identificando los indicadores que guían el mejoramiento de los niveles de vida de la población, observando mejoras significativas. Confirma que existe una relación directa y significativa entre la gestión del presupuesto por resultados y la calidad del gasto; principalmente con los indicadores de programas de Saneamiento Urbano y Rural, Programa de Acceso y Uso adecuado de Servicios de Telecomunicaciones, y finalmente, el Programa de Logros de Aprendizaje de EBR (Educación Básica Regular).

La evaluación de estrategias de gestión para mejorar la administración de los recursos públicos en la municipalidad distrital de Lagunas - Provincia de Alto Amazonas 2021, ha sido validada por expertos, y los 5 expertos concuerdan en que las estrategias en la administración de los recursos públicos en la municipalidad, es acertado, tanto en su concepción teórica como en los resultados de su aplicación. En ese sentido, Guaycha \& Ordóñez (2020), concluye que la calidad, cantidad de la data y el acceso democrático a la información está supedita a la voluntad política. A su vez, Ruano \& Herrero-Gutierrez (2020), indica que la transparencia se da cada vez con mayor frecuencia en España, sobre 
todo desde el momento en el que se empezó a legislar sobre esta realidad, aunque aún quedan aspectos que pueden ser mejorados. Por otro lado, Toro-García et al. (2020), Se busca identificar los aspectos y tendencias más importantes de las nuevas tecnologías aplicadas a las entidades gubernamentales, así como las implicaciones en cuanto a la toma de decisiones y a la mejora de los servicios que el Gobierno presta a sus ciudadanos, concluyendo que es posible afirmar que la influencia y adopción del gobierno digital en diferentes países contribuye a acercar a los ciudadanos con el Estado, aumentado la transparencia y mejorando los servicios. En esa misma dirección, (Paricio-Esteban et al., 2020), el modelo más utilizado por los ayuntamientos es la sede electrónica, siendo el que peores niveles de cumplimiento registra para todos los públicos. La web independiente y el apartado en el sitio web, que son las fórmulas más utilizadas en los ayuntamientos de más de 50.000 habitantes, favorecen más la relación con los ciudadanos y asociaciones locales. Los medios y los órganos de participación son los públicos menos atendidos desde una perspectiva de las Relaciones Públicas en todos los modelos. En el análisis de las regresiones lineales, se evidencia que la web independiente y el apartado en web son significativos en el conjunto de la población, junto con el primer modelo y las plantillas de la Diputación en ayuntamientos más pequeños. El uso de las últimas es una oportunidad para facilitar las relaciones con los stakeholders locales en los municipios de menos de 20.000 habitantes, que cuentan con menos recursos para la elaboración de diseños propios. Por su parte, Valeriano (2019), en materia política se incrementa el factor riesgo país, desacelerando y desalentando las inversiones extranjeras y nacionales, motor de la economía del país. En materia económica, la involución del factor más importante de la macro economía, como es el PBI (Producto Bruto Interno) y a pesar que la actual coyuntura económica la ha estabilizado en el ejercicio 2018, cerrando con $3.8 \%$, continua con visos de no evolucionar por los problemas coyunturales y estructurales del país en todos sus sectores productivos y de servicios. En materia social, el fenómeno del niño costero, el decrecimiento de la población económicamente activa (PEA), y la migración extranjera, que en su gran mayoría no es mano de obra calificada, han incrementado las poblaciones vulnerables (pobreza y extrema pobreza). A su vez, Charry (2018), encontró que la percepción del clima organizacional es desfavorable. También precisan una correlación significativa entre la comunicación interna y las dimensiones: grado de estructura y su influencia en el cargo; consideración, entusiasmo y apoyo; orientación 
hacia la recompensa y orientación hacia el desarrollo y promoción en el trabajo. Finalmente, el 56.5\% refiere que predomina la comunicación interna ineficaz.

\section{CONCLUSIONES}

\section{Se arribó a las siguientes conclusiones:}

1. Se encontró que existe un nivel aceptable de planificación en la dirección y resguardo de los recursos públicos, que está presente en el 63.30\% (19) de los empleados de la municipalidad distrital de Lagunas - Provincia de Alto Amazonas 2021, en la participación se encontró un nivel aceptable en el 66.70\% (20) y el 3.30\% (01) señalan un nivel deficiente. En relación a la eficiencia y capacidad de gasto en la administración de recursos públicos, existe un nivel aceptable en el 50\% (15), a su vez nos muestra que el $13.30 \%$ (04), señalan que la capacidad de gasto es deficiente.

2. Se observa que existe gestión en ejecución presupuestaria en nivel regular, en un $60 \%$ (18), a su vez nos muestra que el 10\% (03) se encuentra en un nivel malo. En la gestión en ejecución de proyectos de inversión, observamos un nivel regular en el $70 \%$ (21) y el 20\% (06) presenta un nivel excelente. La gestión en el gobierno local, se observa que el $73 \%$ es de nivel regular, y el $23.30 \%$ (07) manifiestan un nivel excelente. En la gestión en consulta de deuda, se encontró que el nivel regular presenta un porcentaje elevado que es igual a $70 \%$ en la municipalidad de Lagunas Provincia de Alto Amazonas.

3. La evaluación de estrategias de gestión para mejorar la dirección y resguardo de los recursos públicos en la municipalidad distrital de Lagunas - Provincia de Alto Amazonas 2021, ha sido validada por los expertos, con significación estadística de 95\% y los 5 expertos concuerdan en que las estrategias en la administración de los recursos públicos en la municipalidad, es acertado, tanto en su concepción teórica como en los resultados de su aplicación.

\section{RECOMENDACIONES}

\section{Se propone las siguientes recomendaciones.}

1. Implementación de estrategias de gestión para mejorar la administración de los recursos públicos en la municipalidad distrital de Lagunas - Provincia de Alto Amazonas 2021; debido a su posibilidad de sostenimiento y coherencia que proporcionaran las condiciones para que el proceso de gestión asegure condiciones de 
mayor transparencia en el gasto público, mejorando con ello, la calidad de vida de los pobladores del distrito de Lagunas.

2. Promover acciones de capacitación en los trabajadores municipales que permitan involucrar y comprometerse en el trabajo operativo y de apoyo en el uso de estrategias de gestión, con el propósito de generar mejores condiciones para el control del gasto público y con ello propiciar mayor confianza y credibilidad en la gestión municipal.

3. Establecer acciones de fortalecimiento de capacidades en trabajadores municipales, para propiciar la participación en la elaboración de planes y presupuestos de proyectos de inversión municipal, que permita posibilidades de mejora en lo que refiere a calidad de vida para la población en general y evaluar los proyectos de desarrollo comunal, que permita reconocer las ventajas de las estrategias de gestión local.

\section{7.- REFERENCIAS}

Aceh, kue tradisional khas, \& kue tradisional khas Aceh. (2020). La administración del recurso humano en entidades oficiales del sector de servicios públicos. In Universidad Militar Nueva Granada.

Aguiar Diaz, A., León Santos, M., \& Torres Ponjuán, D. (2020). Auditoría del conocimiento en gobiernos locales. Alcance. Revista Cubana de Información y Comunicación, 9(23), 220-232.

Alarcón Barrero, R., Salvador Hernández, Y., \& Pérez Pravia, M. (2020). Dimensiones Claves De Una Gestión Pública Efectiva En Gobiernos Locales. Aplicación En Un Caso De Estudio. Revista Cientfica de La Universidad de Cienfuegos, 12(6), 411-420.

Benítez-eyzaguirre, L. (2021). Blockchain para la transparencia, gestión pública y colaboración. Teknokultura. Revista de Cultura Digital y Movimientos Sociales, 18(1), 23-32.

Caceres Barrutia, L. E. (2021). Gestión humanista de la administración pública para el fomento de la inteligencia emocional. Ciencias Empresariales, 2, 8-18.

Capello, M., Toselli, V., \& Gonzáles, P. (2020). Transparencia en administraciones municipales de Argentina en 2020. Ieral. Fundación Mediterraneo, 24(199).

Cardenas Pazos, L. L. (2019). Estrategias de gestión municipal y su incidencia en el desarrollo organizacional de la municipalidad provincial de Otuzco 2018. In Universidad Privada de Trujillo. http://hdl.handle.net/20.500.12590/16512 
Castro Premieri, M. E. (2020). Las estrategias de desarrollo municipal, instrumento clave para la gestión local del patrimonio cultural. Arquitectura y Urbanismo, XLI(3), $67-75$.

Cazzolli, A., Echavarria, C., \& Quevedo, C. (2021). Communication strategies from Open Government devices in the province of Córdoba ( Argentina ) Introducción Desde hace dos décadas se han de. Paakat. Revista de Tecnología y Sociedad, 10(19).

Charry Condor, H. O. (2018). The management of internal communication and the organizational climate in the sector public. Comunicacción, 9(1).

Fernández Llera, R. (2020). Buen gobierno local y rendición de cuentas en España. Retos, 10(19), 29-44. https://doi.org/10.17163/ret.n19.2020.02

Fernandez Ramos, S. (2018). La Transparencia Pública: Pasado, presente y futuro. Revista Aragonesa de Administración Publica, 51, 213-243.

Flores de la Cruz, E. Y. (2020). Gestión Política y Administración de los recursos públicos en los Gobiernos Locales de Ancash, 2019. In Universidad Cesar Vallejo (Vol. 4, Issue 1). https://pesquisa.bvsalud.org/portal/resource/en/mdl20203177951\%0Ahttp://dx.doi.org/10.1038/s41562-020-0887-

9\%0Ahttp://dx.doi.org/10.1038/s41562-020-0884-

z\%0Ahttps://doi.org/10.1080/13669877.2020.1758193\%0Ahttp://sersc.org/journ als/index.php/IJAST/article

Flores Vásquez, K. V. (2021). Mejora continua y transparencia de gestión en la Municipalidad distrital de Morales, 2020. Universidad César Vallejo, 0-3. http://repositorio.uncp.edu.pe/bitstream/handle/UNCP/3000/Silva

Acosta.pdf?sequence $=1 \&$ isAllowed=y\%0Ahttps://repositorio.comillas.edu/xmlu i/handle/11531/1046

Guaycha, M., \& Ordóñez, K. (2020). La cultura de datos abiertos en América Latina y la promoción de la Transparencia y Acceso a la información. Revista Ibérica Der Sistemas e Tecnologias de Informacao, 302-314.

Guzmán Vásquez, N. L. (2020). Gestión municipal y estrategias frente al Covid - 19 en la municipalidad distrital de La Banda de Shilcayo, 2020. In Universidad C'ésar Vallejo. $\quad$ http://repositorio.uncp.edu.pe/bitstream/handle/UNCP/3000/Silva Acosta.pdf?sequence=1\&isAllowed=y\%0Ahttps://repositorio.comillas.edu/xmlu i/handle/11531/1046 
Hernandez Sampieri, R., Fernández Collado, C., \& Baptista Lucio. Pilar. (2014). Metodologia de la investigación (R. Hernandez Sampieri (ed.); Sexta edic). Mc Graw Hill Education.

Hurtado de Barrera, J. (2000). Metodología de la investigación holística (Fundación Sypal (ed.); 3a edición). Instituto Universitario de Tecnología Caripito y Servicios de Proyecciones para América Latina.

Juarez Díaz, J. R. (2014). Diseño de investigación en ciencias sociales (Primera).

Khasiani, K., Koshima, Y., \& Mfombouot, A. (2020). Controles de la ejecución presupuestaria para mitigar el riesgo de corrupción en el gasto por la pandemia. Finanzas Públicas, 1-11.

Labandeira, R. (2020). La transparencia y la rendición de cuentas como elementos de innovación en los municipios catalanes : Estándares de calidad en el periodo 2017 - 2018. Gigapp, 7(141), 23-40.

Llanes Font, M. (2018). Convergencia de la ética pública y los objetivos de desarrollo sostenible: resorte subjetivo valioso. Dilemas Contemporáneos, 9(1), 1-11. http://dx.doi.org/10.1016/j.neuropsychologia.2015.07.010\%0Ahttp://dx.doi.org/ 10.1016/j.visres.2014.07.001\%0Ahttps://doi.org/10.1016/j.humov.2018.08.006 \%0Ahttp://www.ncbi.nlm.nih.gov/pubmed/24582474\%0Ahttps://doi.org/10.101 6/j.gaitpost.2018.12.007\%0Ahttps:

Machín Hernández, M. M., Sánchez Vignau, B. S., \& Rodríguez, M. L. (2020). Análisis y proyecciones de la gestión de los servicios públicos en el entorno local. Economía y Desarrollo, 163(1), 1-19.

Martínez Valdés, Y., \& Villalejo García, V. M. (2018). La gestión integrada de los recursos hídricos: una necesidad de estos tiempos. Ingeniería Hidráulica y Ambiental, 39(1), 58-72.

Mendoza-Zamora, W. M., García-Ponce, T. Y., Delgado-Chávez, M. I., \& BarreiroCedeño, I. M. (2018). El control interno y su influencia en la gestión administrativa del sector público. Dominio de Las Ciencias, 4(4), 206. https://doi.org/10.23857/dc.v4i4.835

Mezarina Mendoza, J. P. I., Montenegro Muñoz, S. I., \& Carrasco Freitas, M. D. C. (2021). Diseño y validación de un instrumento para medir el nivel de conocimientos en bioseguridad de odontólogos en tiempos de COVID-19. Revista 
Odontológica $\quad$ Basadrina, 5(1), 19-30. https://doi.org/10.33326/26644649.2021.5.1.1084

Molina Rodríguez-Navas, P., Simelio Solà, N., \& Rodríguez Breijo, V. (2018). Las administraciones locales como vigilantes del cumplimiento de la Ley de Transparencia por las entidades sin ánimo de lucro. Análisis del caso del Ayuntamiento de Barcelona. Revista General de Información y Documentación, 28(1), 61-93. https://doi.org/10.5209/rgid.60811

Moncayo-Vives, G. (2020). Neoinstitucionalismo, transparencia y Gobierno electrónico: Calidad de vida en Bogotá y otras ciudades colombianas. Sarance, 45, 118-142. https://doi.org/10.51306/ioasarance.2020.045.08

Montes Vázquez, A. M. (2020). Análisis del tema de la Transparencia en el Plan Nacional de Desarrollo de los gobiernos de Felipe Calderón Hinojosa (2007-2012) y de Enrique Peña Nieto (2013-2018). Bnemérita Universidad Autónoma de Puebla, 53(9), 1689-1699.

Paricio-Esteban, P., Bruno-Carlos, T., Alonso-Romeero, E., \& García-Alcober, M. P. (2020). Webs y portales de transparencia para la participación ciudadana en la gestión de las relaciones públicas con los stakeholders locales. El Profesional de La Información, 1-17. https://doi.org/10.3145/epi.2020.may.35

Pérez, C. R., \& Carreño, M. (2019). Gestión del gobierno electrónico en las alcaldías. Gestión y Desarrollo Libre, 14.

Quispe De los Santos, J. L., Ramos Giribaldi, A. M., Silva Narvaste, B., \& Llerena Rodríguez, S. Y. (2020). Modelo de open government para optimizar la gestión municipal del gobierno local de Jayanca. Journal of Business and Entrepreneurial, $28(2), 1-43$.

Quispe De los Santos, L. J., Molina Carrasco, Z. C., Quispe De los Santos, J. M., Ramos Gonzales, A. A., \& Hernández Fernández, B. (2019). Open Government, otra visión en la Gestión Municipal. In Compas.

Quispe, V., Villaroel, R., Copaja, F., \& Santa María, H. (2021). Gestión por resultados y la calidad del gasto en el sector público. In Compas. http://142.93.18.15:8080/jspui/bitstream/123456789/670/3/gestión por resultados y calidad del gasto_CORREGIDO.pdf

Ríos Cázares, A., \& Cejudo, G. M. (2020). El acceso a la información gubernamental: 
análisis empírico de políticas de transparencia en cuatro países centroamericanos. Revista de Gestión Pública, 2(2), 335. https://doi.org/10.22370/rgp.2013.2.2.2314

Rivera Valles, H. (2020). Modelo de gestión según herramienta de transparencia en la ejecución presupuestaria de la provincia Mariscal Cáceres, 2019. In Universidad C'ésar Vallejo. http://repositorio.uncp.edu.pe/bitstream/handle/UNCP/3000/Silva Acosta.pdf? sequence $=1 \&$ isAllowed=y\%0Ahttps://repositorio.comillas.edu/xmlu i/handle/11531/1046

Robles-López, C. M., \& Zamora-Medina, R. (2020). Online transparency as an intangible asset of the public sector. Transinformacao, 32. https://doi.org/10.1590/16789865202032e190059

Rodríguez-Martín, A., Palomo-Zurdo, R., \& González-Sánchez, F. (2020). Transparency and circular economy: Analysis and assessment of municipal management solid waste. CIRIEC-Espana Revista de Economia Publica, Social y Cooperativa, 99, 233-272. https://doi.org/10.7203/CIRIEC-E.99.16011

Rodriguez Panduro, M. S., Palomino Alvarado, G. del P., \& Aguilar Saldaña, C. M. (2020). Eficiencia, eficacia y transparencia del gasto público municipal. Ciencia Latina Revista Científica Multidisciplinar, 2215(2), 704-719. https://doi.org/10.37811/cl_rcm.v4i2.108

Romani, R. (2021). Modelo de Gestión de Recursos Humanos basado en competencias implementado en ambitos publicos. Universidad Tecnológica Nacional Concepción Del Uruguay.

Ruano López, S., \& Herrero-Gutierrez, F.-J. (2020). Transparencia politica y económica de las corporaciones municipales. Las webs de los ayuntamientos de las localidades más pobladas de Madrid. Inclusiones, 7, 1689-1699.

Sánchez Carlessi, H., Reyes Romero, C., \& Mejía Sáenz, K. (2018). Manual de términos en investigación científica, tecnológica y humanística (Universidad Ricardo Palma (ed.); Primera ed).

Sandoval Carranza, M. A. (2018). Ética pública en el uso de los recursos públicos en la Municipalidad Distrital de Piás, 2017. Universidad Cesar Vallejo.

Secretaría de Asuntos Políticos, Departamento para la Gestión Pública Efectiva, \& Organización de los Estados Américanos. (2012). Guía de Estrategias y 
Mecanismos para la Gestión Pública Efectiva (GEMGPE) Perú. Www.Oas.Org.

Suárez, E. (2020). Transparencia administrativa y derecho de acceso a la información administrativa. Alcance. Revista Cubana de Información y Comunicación, 9(23), $22 \mathrm{p}$.

Sue González, A., Herrera, M. E., \& García, E. (2021). Algunas estrategias para el fortalecimiento de los controles internos como medios para evitar los malos manejos de los bienes públicos. Revista Cathedra, 15, 11-25. https://doi.org/10.37594/cathedra.n15.469

Tejedo-Romero, F., \& Ferraz Esteves Araujo, J. F. (2018). Transparencia en los municipios españoles: determinantes de la divulgación de información. $\begin{array}{llll}\text { Convergencia Revista de Ciencias } & \text { Sociales, }\end{array}$ https://doi.org/10.29101/crcs.v25i78.9254

Toro-García, A. F., Gutiérrez-Vargas, C. C., \& Correa-Ortiz, L. C. (2020). Estrategia de gobierno digital para la construcción de Estados más transparentes y proactivos. Trilogía Ciencia Tecnología Sociedad, 12(22), 71-102. https://doi.org/10.22430/21457778.1235

Tumi Quispe, J. E. (2020). Rendición de cuentas en la gestión del gobierno municipal de Puno-Perú (2011-2018). Comuni@cción: Revista de Investigación En Comunicación y Desarrollo, 11(1), 63-76. https://doi.org/10.33595/22261478.11.1.393

Valeriano Ortiz, L. F. (2013). La Gestión Pública Del Perú. Rev. de Investigación de La Fac. de Ciencias Administrativas, 16(31), 73-79.

Valeriano Ortiz, L. F. (2019). Transparencia en la gestión pública y privada del Perú frente a la crisis de valores que afectan el desarrollo del país. Gestión En El Tercer Milenio, 22(43), 31-43.

Vargas Merino, J. A., \& Zavaleta Chávez, W. E. (2020). La gestión del presupuesto por resultados y la calidad del gasto en gobiernos locales. Visión de Futuro, 24(24, No 2

(Julio-Diciembre)), $37-59$. https://doi.org/10.36995/j.visiondefuturo.2020.24.02.002.es

Vargas, S. (2017). Maltrato Infantil en la familia y la conducta emocional en niñas y niñas. Universidad Nacional Del Altiplano. Universidad Nacional Del Altiplano, $1-53$. 
http://tesis.unap.edu.pe/bitstream/handle/UNAP/7786/Vargas_Cardenas_Sulbi_ Diana.pdf? sequence $=1 \&$ isAllowed $=\mathrm{y}$

Velasco Sánchez, E. (2020). ¿Puede la nueva gestión pública fortalecer la transparencia gubernamental?: el caso de la gestión del desempeño. Revista de Gestión Pública, 2(2), 383. https://doi.org/10.22370/rgp.2013.2.2.2315

Vinueza-franco, J. I. (2020). La optimización y el control interno en el uso de los recursos públicos en la mejora de la gestión administrativa. FIPCAEC, 5, 14-38.

Zamora-de-Ortiz, M. S., Serrano-Pastor, F. J., \& Martínez-Segura, M. J. (2020). Validez de contenido del modelo didáctico P-VIRC (preguntar-ver, interpretar, recorrer, contar) mediante el juicio de expertos. Formación Universitaria, 13(3), 43-54. https://doi.org/10.4067/s0718-50062020000300043 